\title{
Acute myocarditis secondary to acute Campylobacter jejuni infection
}

\author{
Authors: Graeme Martin Greenfield, ${ }^{A}$ Jonathan Mailey, ${ }^{B}$ Kristopher Lyons ${ }^{C}$ and Thomas Graham Trouton ${ }^{D}$
}

We report a case of myocarditis complicating gastroenteritis caused by Campylobacter jejuni. The patient had an uncomplicated course and made a full recovery. Cardiac MRI was useful in securing an early diagnosis and establishing baseline heart function. Myocarditis should be considered in younger patients presenting with chest pain and elevations in serum troponin. The occurrence of myocarditis complicating Campylobacter jejuni gastroenteritis is reviewed.

KEYWORDS: Myocarditis, Campylobacter jejuni, cardiac MRI, troponin

\section{Case presentation}

A 31-year-old male with no relevant past medical history presented to the emergency department with acute onset central chest pain. He described persistent watery diarrhoea with one episode of bloody stool over the preceding week. There were associated symptoms of fever, myalgia and lethargy. He had no vomiting. The night prior to admission he awoke with severe central chest pain. The pain initially eased, but culminated in a severe episode 12 hours later which precipitated his call for medical assistance.

On arrival to the emergency department his observations were recorded as a respiratory rate $=19 / \mathrm{min}$, oxygen saturations $=100 \%$ on room air, heart rate $=73 \mathrm{bpm}$, blood pressure $=143 / 67 \mathrm{mmHg}$, temperature $=36.3^{\circ} \mathrm{C}$, and blood glucose $=5.3 \mathrm{mmol} / \mathrm{L}$.

Examination revealed normal heart sounds with no rubs or murmurs, lung fields were clear to auscultation and his abdomen was soft and non-tender. He had an injected conjunctiva in the right eye with upper lid blepharitis evident. Chest X-ray demonstrated no abnormalities. Electrocardiogram (ECG) showed normal sinus rhythm with no ST segment abnormalities. Full blood count showed a haemoglobin level of $142 \mathrm{~g} / \mathrm{L}$, white cell count of $7.3 \times 10^{9} / \mathrm{L}$ and platelets of $280 \times 10^{9} / \mathrm{L}$. C-reactive protein (CRP) was $52 \mathrm{mg} / \mathrm{L}$.

Initial biochemistry showed sodium $141 \mathrm{mmol} / \mathrm{L}$, potassium 4.4 $\mathrm{mmol} / \mathrm{L}$, urea $5.7 \mathrm{mmol} / \mathrm{L}$ and creatinine $90 \mu \mathrm{mol} / \mathrm{L}$. His D-dimer

Authors: Aacademic clinical fellow, Queen's University Belfast, Belfast, UK; ${ }^{\text {B }}$ specialty trainee year 3 cardiology, Antrim Hospital, Antrim, UK; ' Consultant cardiologist, Antrim Hospital, Antrim, UK; ${ }^{D}$ consultant cardiologist, Antrim Hospital, Antrim, UK was mildly raised at $358 \mathrm{ng} / \mathrm{mL}$. Initial troponin T was raised at $320 \mathrm{ng} / \mathrm{L}$, increasing to $402 \mathrm{ng} / \mathrm{L}$ at 4 hours.

\section{Differential diagnosis}

The initial diagnosis in the Emergency Department was acute coronary syndrome given the presentation of chest pain with associated troponin rise. Given the patient's age and low risk profile for coronary artery disease, myocarditis was also considered as a differential diagnosis. A viral gastroenteritis was felt to be the cause of the diarrhoeal illness, with the possibility of a common pathogen resulting in gastroenteritis and myocarditis.

\section{Initial management and prognosis}

Following the finding of the initial raised troponin, the patient was given a loading dose of aspirin, ticagrelor and therapeutic enoxaparin in the emergency department and referred to cardiology. Acute myocarditis was identified as the more likely diagnosis on cardiology review and ticagrelor and enoxaparin treatment discontinued. Echocardiography confirmed normal LV systolic and diastolic function with no regional wall motion abnormalities, significant valvular abnormalities or pericardial effusion. Supportive care was initiated. Stool culture and polymerase chain reaction (PCR) was requested as well as serology for common viral agents implicated in myocarditis.

\section{Case progression and outcome}

Initial stool cultures flagged positive for Campylobacter $\mathrm{sp}$ on PCR and this was confirmed on subsequent testing. Blood cultures performed during an episode of pyrexia were negative. The presence of Campylobacter jejuni was confirmed with the microbiology team and antibiotics were withheld in view of improving gastrointestinal symptoms.

Cardiac magnetic resonance imaging (MRI) performed during the inpatient stay showed normal LV volumes and function with localised myocardial oedema and contrast enhancement within the basal inferolateral wall consistent with acute myocarditis (Fig 1).

Following a good recovery with supportive care he was discharged home on bisoprolol $2.5 \mathrm{mg}$ once daily. A take out sandwich was identified as the most likely source of infection and the public health agency was informed.

At 6-week review he reported total resolution of symptoms and had returned to normal activities and work while abstaining from strenuous exercise. 


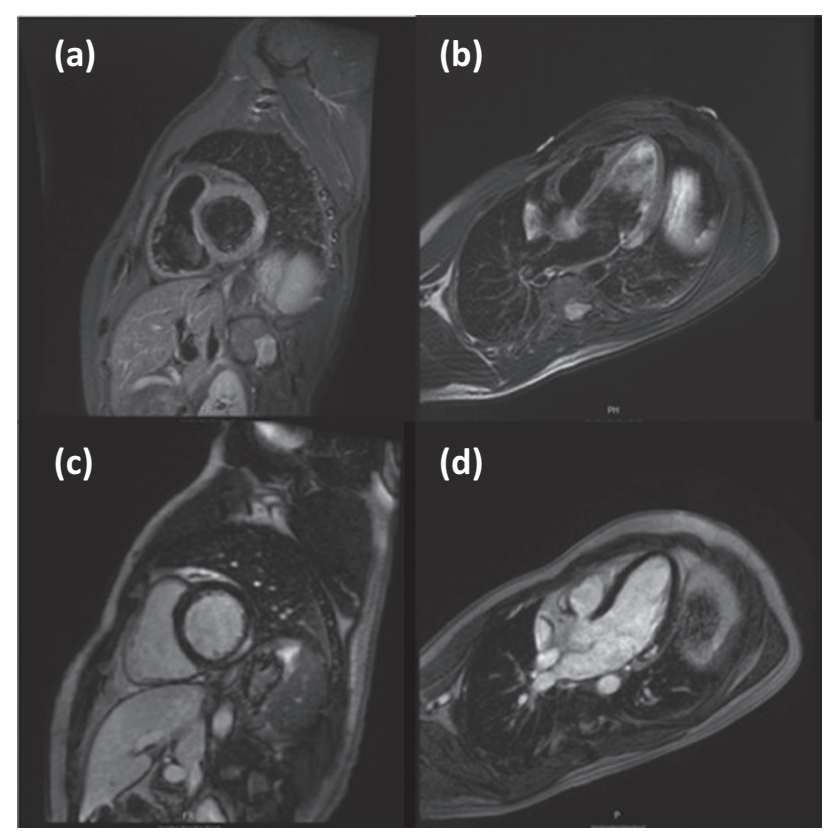

Fig 1. Cardiac MRI. High signal region on STIR imaging within the basal inferolateral wall on (a) short axis and (b) long axis images due to myocardial oedema. (c,d) High signal within the basal inferolateral wall in a midwall and epicardial distribution indicating a degree of myocardial replacement fibrosis. STIR = short inversion time inversion recovery

\section{Discussion}

Acute myocarditis is potentially life threatening and often represents a complex diagnostic challenge. Presentation is varied, with symptoms that often include chest pain and breathlessness but can range from lethargy and malaise to acute heart failure, arrhythmia and sudden death. The aetiology is complex, with viral agents (for example, Coxsackie virus, influenza virus, herpes virus, HIV, cytomegalovirus [CMV] and Epstein-Barr virus [EMV]) representing the primary cause in the developed world. Often the exact viral agent will not be identified. Other causes include bacterial, fungal, rickettsial or protozoan parasite infection, cardiotoxic agents and autoimmune systemic disease. ${ }^{1}$

In the majority of cases the disease is self-limiting, with approximately half of cases showing full resolution by 4-6 weeks. Around one-quarter of cases demonstrate persistent cardiac dysfunction, meanwhile a further quarter progress to long-term dilated cardiomyopathy with a very small proportion requiring advanced heart failure therapies and cardiac transplantation. Biventricular dysfunction is associated with a poorer prognosis. ${ }^{2}$ Exercise should be avoided for 6 months following an episode of myocarditis due to the small risk of potentially fatal arrhythmia. C jejuni is a common cause of food poisoning, with consumption of raw or undercooked meat or poultry, contaminated water or unpasteurised dairy products often the source. Infections are generally self-limiting in immunocompetent patients. The disease commonly presents with abdominal cramps, watery diarrhoea with or without rectal bleeding, fever, rigors and myalgia.
Antibiotic therapy is reserved for severe disease refractory to supportive management. There is a well-documented association with reactive arthritis and Guillain-Barré Syndrome as late complications of the disease process.

This case highlights a rare and potentially life-threatening complication of acute $C$ jejuni infection. The use of cardiac MRI allowed for an accurate diagnosis and avoided the need for invasive coronary angiography to exclude coronary artery disease. Invasive cardiac biopsy was not required as the risks outweighed any potential diagnostic benefit. There was a relatively benign course to this illness with only supportive care required. Acute myocarditis during acute infection with Campylobacter has been rarely reported. Given the relative scarcity of this association, it is not clear whether the inflammation of the myocardium is secondary to an immunological phenomenon, such as that noted in reactive arthritis and Guillain-Barré Syndrome, or as a result of direct action of the bacteria upon cardiac myocytes. There is insufficient information to draw firm conclusions on the prognosis of campylobacter myocarditis and fatal cases have been reported. $^{3}$

\section{Key learning points}

> It is vital to take a thorough history in patients presenting with troponin positive chest pain to elicit more unusual aetiologies

> Myocarditis should be suspected in all troponin positive presentations with coexistent infective symptoms

> Campylobacter infection may be associated with a concurrent myocarditis

> Prognosis in myocarditis is generally good with no current identifiable links between disease severity and causative agent

> Patients presenting with significant myocardial dysfunction are likely to have worse outcomes.

\section{Consent}

The patient provided written consent for publication of this case.

\section{References}

1 Schultheiss HP, Kühl U, Cooper LT. The management of myocarditis. Eur Heart J 2011;32:2616-25.

2 Caforio AL, Pankuweit S, Arbustini E et al. Current state of knowledge on aetiology, diagnosis, management, and therapy of myocarditis: A position statement of the European Society of Cardiology Working Group on Myocardial and Pericardial diseases. Eur Heart J 2013;34:2636-48.

3 Pena LA, Fishbein MC. Fatal myocarditis related to Campylobacter jejuni infection: a case report. Cardiovasc Pathol 2007;16:119-21.

Address for correspondence: Dr Graeme Greenfield, Centre for Cancer Research and Cell Biology, Queen's University Belfast, 97 Lisburn Road, Belfast BT9 7AE, UK.

Email: g.greenfield@qub.ac.uk 